\title{
SYSTEMATIC IN SILICO EXPLORATION OF CHEMICAL SPACE AROUND DRUG CANDIDATE DD217, A POTENT ANTICOAGULANT TARGETING FACTOR XA
}

\author{
D. Shulga' ${ }^{1}$, N. Tserkovnikova ${ }^{2,3}$, D. Tarasov ${ }^{2,3}$, D. Tovbin ${ }^{2,3}$ \\ ${ }^{1}$ Department of Chemistry Dept, Moscow State University, \\ 119991, Russia, Moscow, Leninsky Gory, 1/3. \\ ${ }^{2}$ Department of Kinetics and Catalysis, Institute of Chemical Physics \\ of Russian Academy of Sciences, 119991, Russia, Moscow, Kosygina str., 4. \\ ${ }^{3}$ PharmaDiall LLC, 121205, Russia, Moscow, Nobel str., 7.
}

DOI: 10.19163/MedChemRussia2021-2021-63

E-mail: shulga@qsar.chem.msu.ru

Recentlya potent factor Xa ( $\mathrm{fXa}$ ) inhibitor with the promising pharmacodynamics, DD217 has been reported [1]. However, the chemical space available for synthesis preserving the same core (Figure) [2] has not been fully explored in order to find out even more potent molecules, or the molecules with more desirable ADMET properties. The main objective of the work is to conduct such exploration using in silico tools.<smiles>[R3]c1ccc(NC(=O)c2cc([R3])cc([R4])c2NC(=O)c2c([R20])c([R6])c(N([R6])[R6])c([R6])c2[R6])c([R2])c1</smiles>

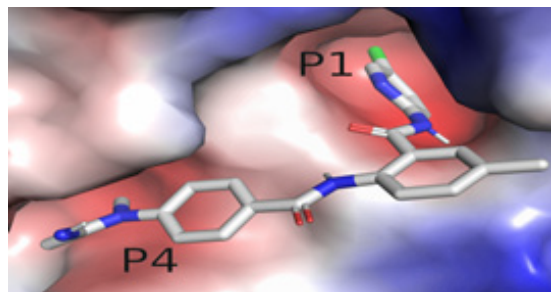

The cardinality of the chemical space available was estimated as $\sim 30 \mathrm{bln}$ structures. Thus a divide-and-conquer approach was adopted for further study, in which only certain combinations of the substituents were studied at once, followed by removal of the worst and proceeding with the other substituents. Pose generation (using fXa target from PDB:1IQI) and scoring was done using AutoDock, which appeared superior for this specific task compared to AutoDock Vina. The variation of chemical substituents was explained in terms of Free-Wilson like QSAR models.

The revealed influence of the substituents estimated in the work is in good accord with the known SAR data for the ligands of fXa target and found to be well explainable based on analysis of ligand-receptor interactions implied for the generated docking positions. Thus, the results will help guide further development.

\section{References}

[1] D. Tarasov, D. Tovbin, D. Malakhov et al. // Current Drug Discovery Technologies, 2018, 15, $335-50$.

[2] Patent PCT/RU2011/000129 (02.03.2011). 